
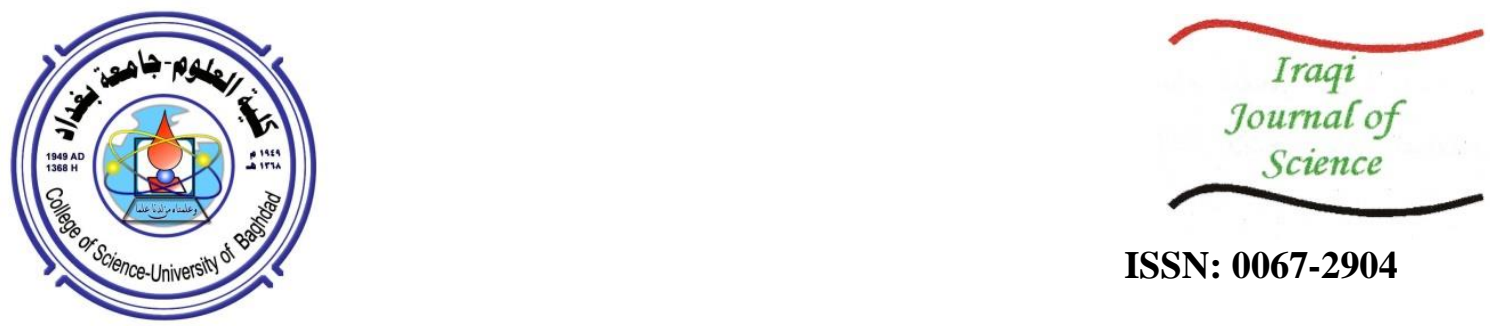

ISSN: 0067-2904

\title{
Qualitative and Quantitative Studies of the Effects of Spherical Aberration on the Optical System in the Cases of Coherent and Incoherent Illumination
}

\author{
Hassan Hammadi*, Ayad A. AL-Ani \\ Department of Physics, College of Science, AL-Nahrain University, Baghdad, Iraq \\ Received: 12/1/2020 \\ Accepted: $30 / 4 / 2020$
}

\begin{abstract}
In this paper, we study the effects of coherent and incoherent illumination on the optical imaging system. The effects were studied qualitatively in terms of Point Spread Function (PSF) and Modulation Transfer Function (MTF), and quantitatively in terms of Root Mean Square Error (RMSE). Different values of radius of aperture were investigated in the presence and absence of spherical aberration with various magnitudes of spherical aberration $(\mathrm{M}=1,2$, and 3$)$. The experiments were performed using homogeneous media.

The results show that imaging with incoherent illumination is better than imaging with coherent illumination, especially for small aperture. Also, we found that the effects of spherical aberration were increased under incoherent illumination and large aperture.
\end{abstract}

Keywords: Point Spread Function (PSF), Modulation Transfer Function (MTF), spherical aberration, Coherent and incoherent illumination

\section{دراسة نوعية وكمية لتأثير الزيغ الكروي على النظام البصري في حالة الإضاءة المتثاكهة وغير المتثاكهة}

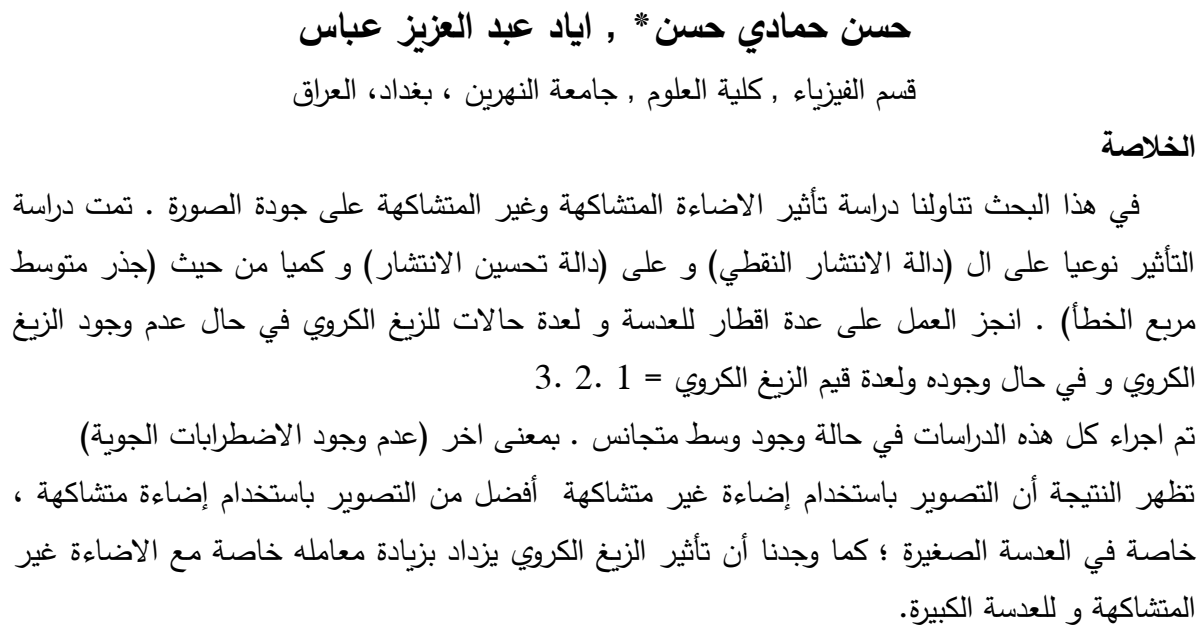

*Email: hasansaw61@Gmail.com 


\section{Introduction}

The Point Spread Function (PSF) is defined as the energy distributed on the image plane due to a point source located on the object plane [1], For several computer vision apps, the average PSF assessment of the image procurement system is important, as in edge detection and defocus depth. The perfect PSF is the three-dimensional diffraction pattern of light emitted from an infinitesimally small point source throughout the specimen and transmitted to the image plane via a high numerical aperture [2].

PSF was acquired via one-dimensional systems of a pupil filter beneath the spherical wave aberration with antiphase anodization [3].

The optical designers are trying to compare the quality of optical systems; a widely used measure is the MTF modulation transfer function where MTF is the PSF Fourier Transform.

The MTF is an important approach that assists in evaluating optical systems in terms of imageforming capability. Not only does MTF include the means of objectively and quantitatively expressing the image quality of optical systems, but it can also be measured from the data on lens design. Throughout this way, optical and system designers can surely portray the optical systems achievement. The image quality of the produced lenses could be compared with both model standards [4].

By the comparison of two wide-field optical coherence tomography illumination modes, it was found that temporally coherent illumination generates coherent cross-discussion, causing significant image degradation, and that spatially incoherent illumination with an appropriate interferometer model offers an effective mechanism for suppressing coherent cross-discussion [5].

Numerous mechanisms have been utilized to manufacture nanostructured thin films for PSF, such as high aperture [6].

\section{Theory}

Spherical aberration is a kind of aberration found in optical systems using spherical ground aspects. Lenses and parabolic mirrors are often designed of spherical objects, as this shape is easier to establish than curved surfaces that are not spherical. Light rays that hit off-center spherical surfaces are refracted or more or less reflected than those which hit close to the center. This variance reduces the image quality of optical systems [7].

In general, aberration can be described as a departure of the efficiency of paraxial optics from the predictions of an optical system. In an imaging system, it happens after transmission via the system that light from one point of an object does not converge into a single point. Aberrations happen because the easy paraxial theory is not a fully accurate model of the effect of an optical system on light, but rather because of flaws in the optical component [8].

Because a white light source such as a light bulb is small for coherent and incoherent illumination, the filament is regarded to be a spatially incoherent source [5].

The PSF reduces the achievable magnification for a specified magnifying lens [9].

There are other several factors that have similar impacts, such as migration or the movement of new individuals into or out of the population [10].

Let us assume that the light emanating from different elements of the object is incoherent. Consequently, the intensity of the light at a point $\mathrm{x}, \mathrm{y}$ in the image plane $\operatorname{img}(\mathrm{x}, \mathrm{y})$ corresponding to an element $\mathrm{dx} 。 * d y_{\circ}$ centered on an object point $\left(x_{\circ}, y_{\circ}\right)$ ' $o b j(x, y)$ is given by [1]:

$$
\operatorname{img}(x, y)=\iint_{-\infty}^{\bowtie} \text { obj }\left(x_{\circ}, y_{\circ}\right) \operatorname{PSF}\left(x, y ; x_{\circ}, y_{\circ}\right) d x 。 d y 。
$$

Now if the PSF is space invariant or isoplantic, where the physical meaning of the isoplantic is that the PSF is unchanged across the image plane, then eq .1 can be written as [1]:

$$
\operatorname{img}(x, y)=\iint_{-\infty}^{\bowtie} o b j\left(x_{\circ}, y_{\circ}\right) \operatorname{PSF}\left(x_{-} x_{\circ}, y_{-} y_{\circ}\right) d x 。 d y 。
$$

Eq .2 indicates that imaging with incoherent illumination and space invariant PSF is just a convolution of the object with the PSF

$$
\operatorname{img}(x, y)=\operatorname{obj}\left(x_{\circ}, y_{\circ}\right) \otimes \operatorname{PSF}(x, y)
$$

where $\otimes$ denotes convolution process where the convolution is a mathematical way of combining two signals to form a third one. It is the single most important technique in Digital Signal Processing. [11] 
The PSF for incoherent illumination can be represented by [1]

while for coherent illumination

$$
\operatorname{PSF}(\mathbf{x}, \mathbf{y})=|\mathbf{F T}(\operatorname{pup}(\mathcal{x}, \mathbf{v}))|^{2}
$$

$$
\operatorname{PSF}(x, y)=|F T(\operatorname{pup}(\mathcal{x}, v))|
$$

The modulation transfer function (MTF) was evaluated from eqs 4 and 5 and for the two cases of illumination (coherent and incoherent) [12]

where $|\ldots$.$| represents the Modulus of the function.$

$$
\operatorname{MTF}(x, y)=\mid \text { FT }(\operatorname{PSF}(\mathcal{X}, v)) \mid
$$

FT denotes the Fourier transform and $\operatorname{pup}(\boldsymbol{\varkappa}, \boldsymbol{v})$ define the pupil function.

$\mathcal{H}, \boldsymbol{v}$ represents the spatial frequency coordinate.

Now, when there is no aberration, $\operatorname{pup}(\boldsymbol{x}, \boldsymbol{v})$ is given by [1].

where $\mathrm{R}$ is the radius of the pupil function.

$$
\operatorname{pup}(\mathcal{X}, v)=\left\{\begin{array}{l}
1, \text { if }\left(\mathcal{X}^{2}, v^{2}\right) \leq R^{2} \\
0, \text { otherwise }
\end{array}\right.
$$

Now, In the presence of aberration, such as spherical aberration, coma, astigmatism or defocusing error, the pupil function can be presented by [13].

$$
\operatorname{pup}(\varkappa, v)=\left\{\begin{aligned}
\mathbf{e}^{\mathrm{i} \theta(\varkappa, v)}, & \text { if }\left(\varkappa^{2}, \mathbf{v}^{2}\right) \leq \mathbf{R}^{2} \\
0, & \text { otherwise }
\end{aligned}\right.
$$

where $(\boldsymbol{\theta})$ is the phase difference in the pupil plane due to the aberration error. The phase difference function is different for each type of aberration. Spherical aberration for the phase difference, $\theta_{\mathrm{sph}}(\boldsymbol{\mathcal { H }}$,$) ,$ is given by [13]:

$$
\boldsymbol{\theta}_{\mathrm{sph}}(\boldsymbol{x}, \boldsymbol{v})=\left(\mathbf{M}_{\mathrm{sph}} \mathbf{a}^{4}\right) \frac{2 \pi}{\lambda}
$$

where " $\mathrm{M}_{\mathrm{sph}}$ " is the spherical aberration coefficient representing the magnitude of spherical aberration and "a" is the normalized distance of each point in the pupil plane from the center of the plane, i.e. a, is given by [1]:

$$
\mathbf{a}^{2}=\frac{\left(\mathcal{x}-\mathfrak{x}_{\mathbf{c}}\right)^{2}+\left(\mathbf{v}-\mathbf{v}_{\mathbf{c}}\right)^{2}}{\mathbf{R}^{2}}
$$

where $\left(\varkappa_{c}, \cup_{c}\right)$ are the coordinates of the center point of the pupil plane.

\section{Numeric Results and Discussion}

The study of the effects of coherent and incoherent illumination on PSF and MTF in the cases of absence and existence spherical aberration was conducted for two different radii of aperture $(\mathrm{R}=10$ pixels and $\mathrm{R}=40$ pixels), simulated in an image of 256x256 size. The effects of spherical aberration were studied for different magnitudes: $M=0$ at no aberration and $M=1,2$ and 3 at different magnitudes of aberration.

First- In the case of no aberration, the PSF and MTF for a certain pupil function radius were simulated as follows:

a- We generated a circular function ( representing the pupil function) with a certain radius $\mathrm{R}$ as in eq (7).

b- In the case of coherent and incoherent illumination, we calculated the PSF and MTF using eq (4) and (5) and MTF using eq (6).

Second- In the case of the presence of spherical aberration, the PSF and MTF were evaluated in the same way as in the above procedure, except that when we generated a circular function, we used eq (8) and eq (9) instead of eq (7), and for certain values of spherical aberration coefficient (M).

All the results were evaluated by writing a related program using matlab-software.

Figure-1 and Figure-2 represent the PSF in the cases of coherent and incoherent illumination for apertures with radii of $R=10$ and 40 , respectively. 


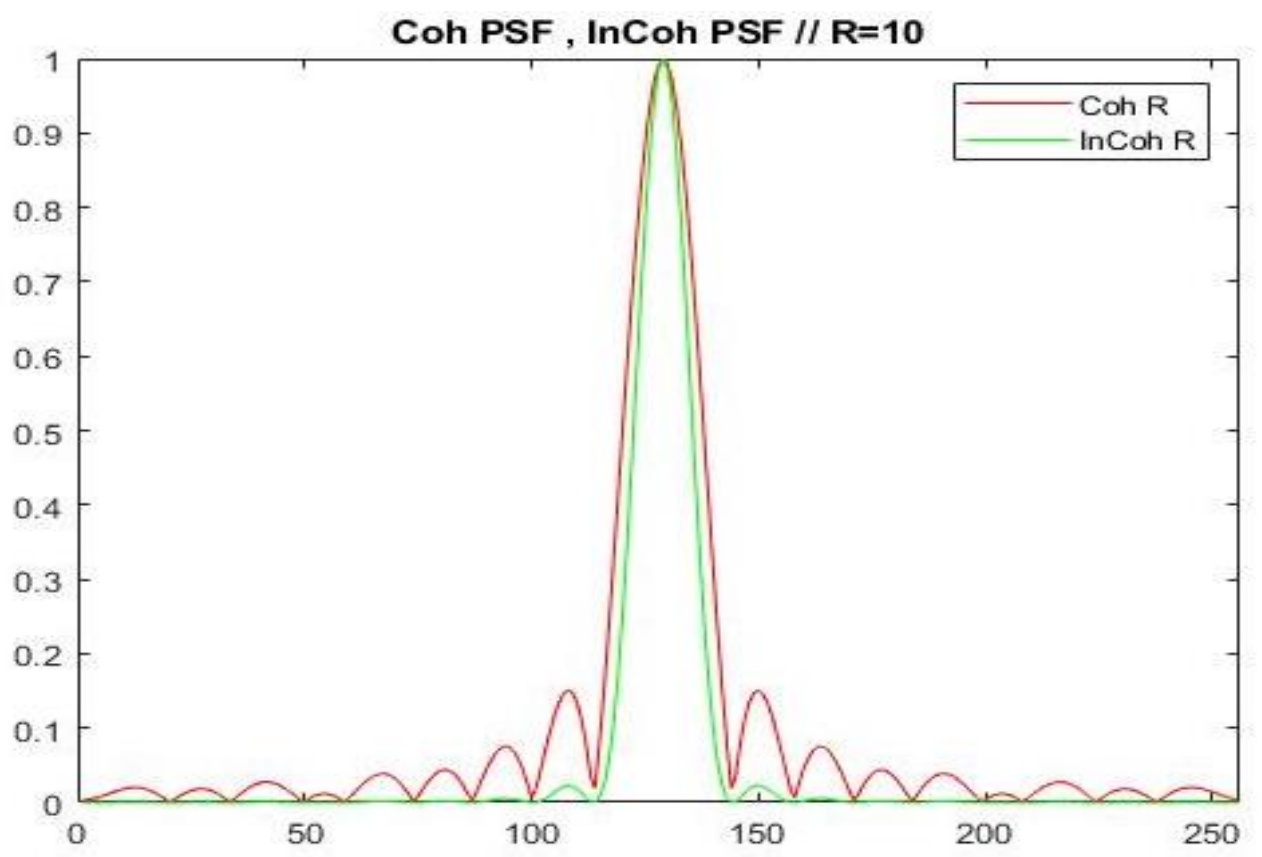

Figure 1- The PSF of the optical system in the case of coherent and incoherent illumination when $\mathrm{R}=10$

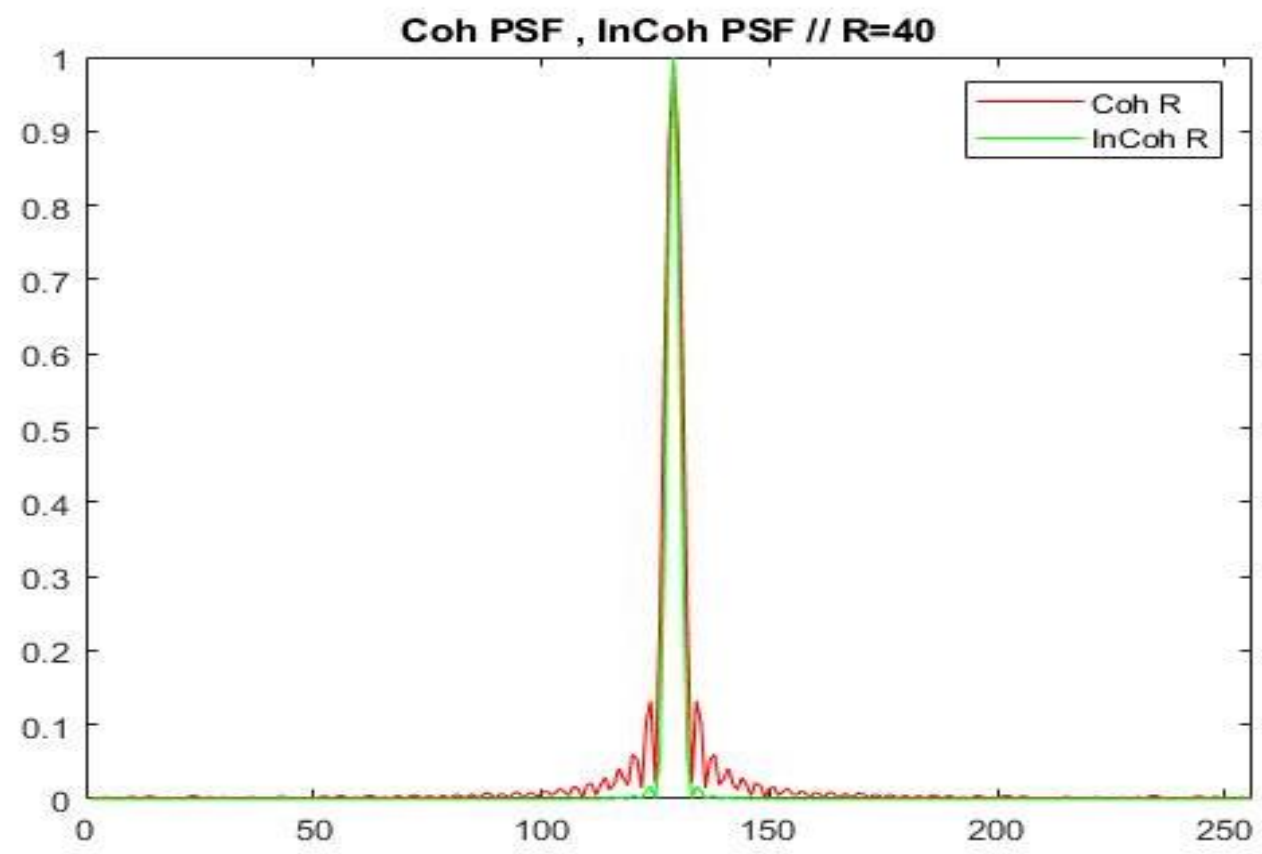

Figure 2-The PSF of the optical system in the case of coherent and incoherent illumination when $\mathrm{R}=40$.

From Figures-(1, 2), we found that the PSF becomes narrower when R is large for the two types of illumination (coherent and incoherent), which indicates a better optical imaging system.

Figure- 3 and Figure- 4 represent the MTF in the cases of coherent and incoherent illumination for apertures with radii of $\mathrm{R}=10$ and 40 , respectively. 


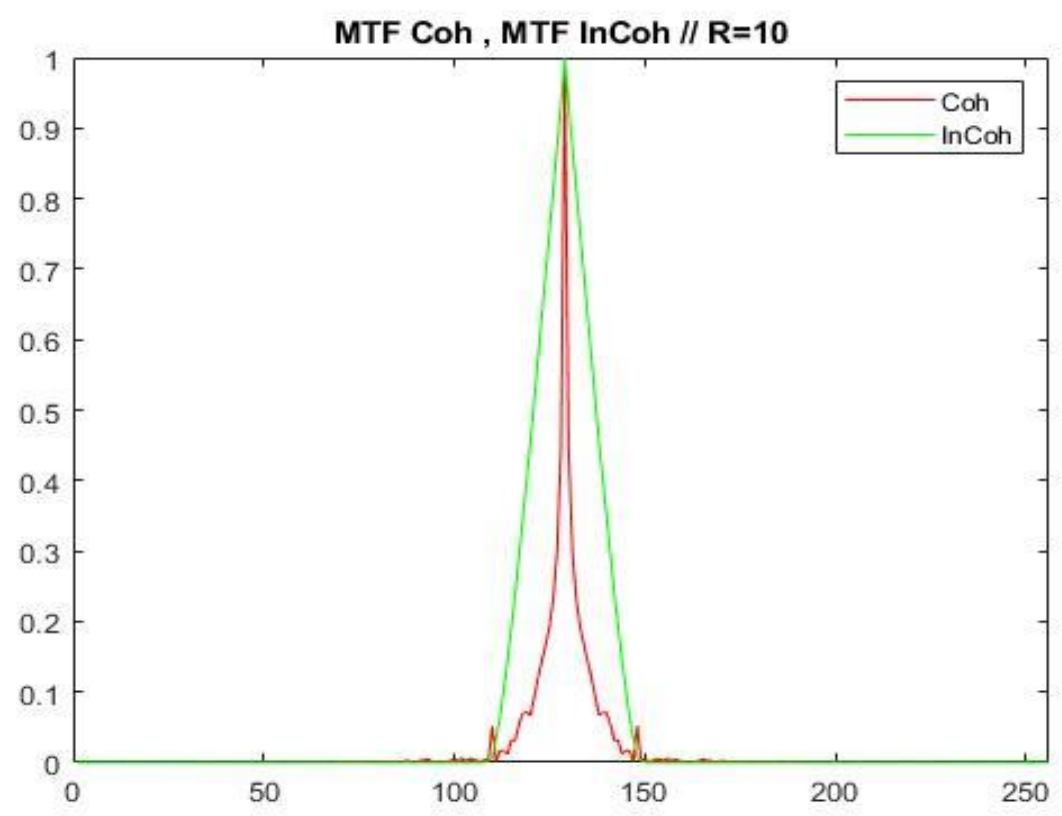

Figure 3-The MTF of the optical system in the case of coherent and incoherent illumination when $\mathrm{R}=10$

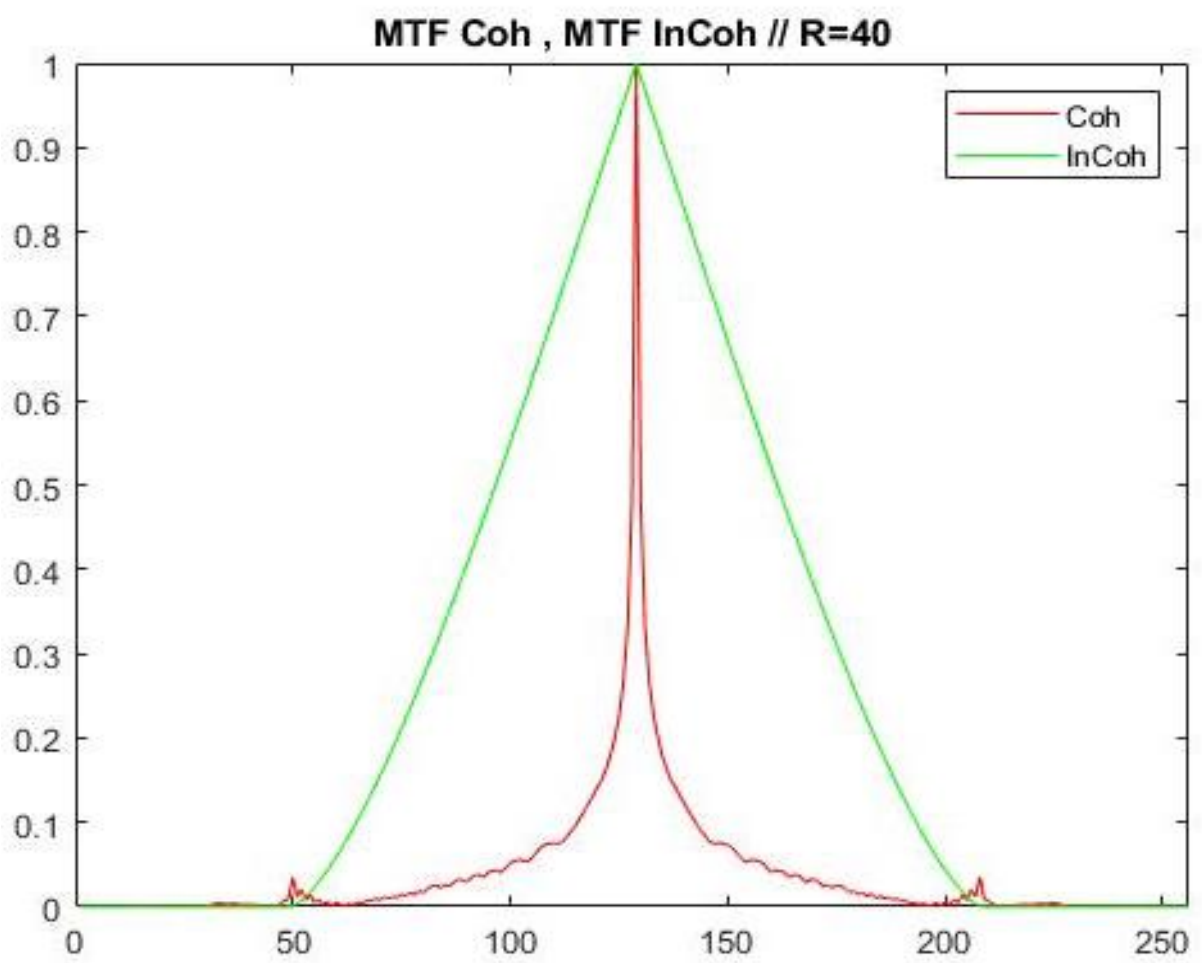

Figure 4-The MTF of the optical system in the case of coherent and incoherent illumination when $\mathrm{R}=40$

From Figures- (3) and (4), we found that the MTF becomes wider when R is large for both types of illumination (coherent and incoherent), which implies a better optical imaging system. Also, we found that MTF becomes very bad in the case of coherent illumination, especially when R is increased.

Figure-5 and Figure- 6 represent the effects of different magnitudes of spherical aberration $(M=0,1$, 2 and 3) on Point Spread Function in case of incoherent illumination for apertures with radii of $\mathrm{R}=10$ and 40 , respectively. 


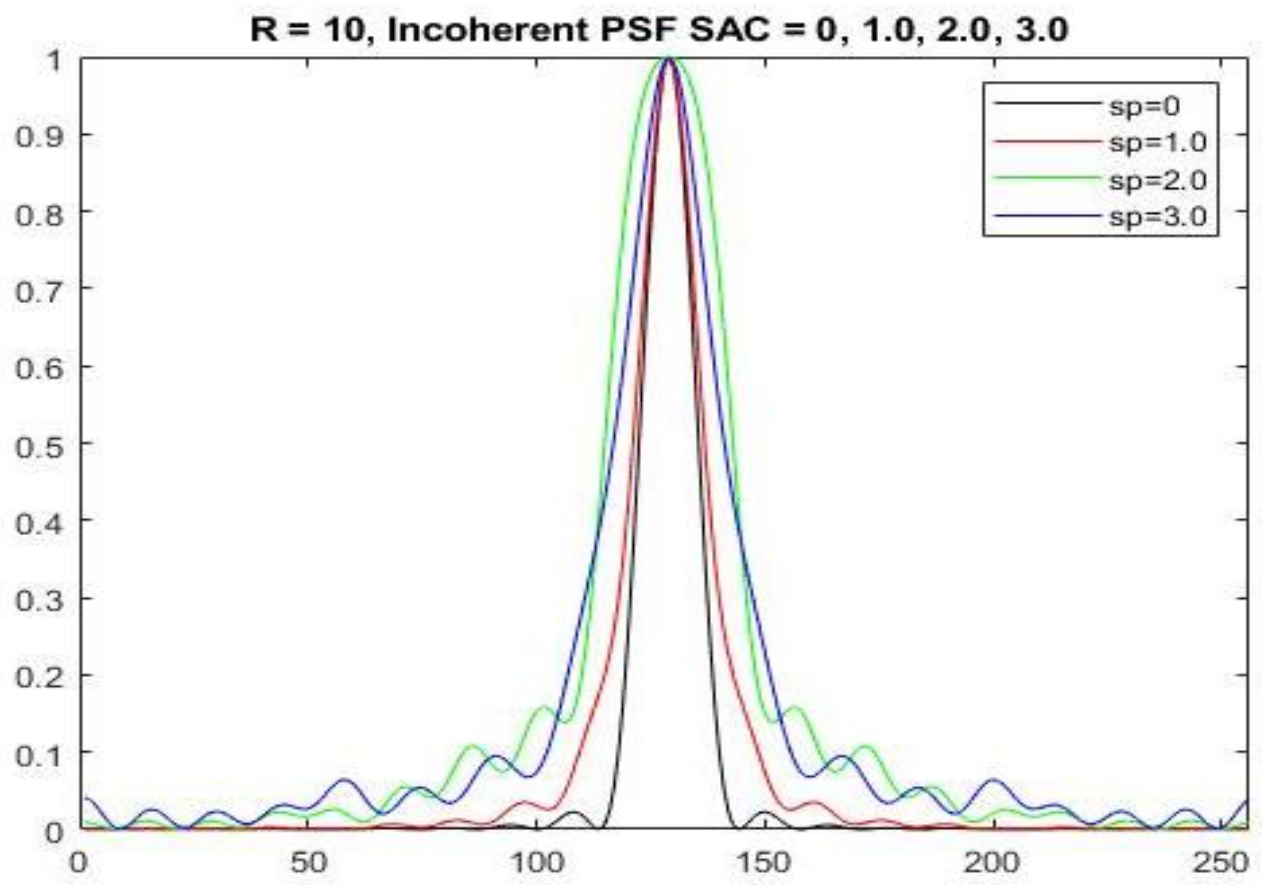

Figure 5-The PSF of the optical system in the case of incoherent illumination in the presence of spherical aberration when $\mathrm{R}=10$

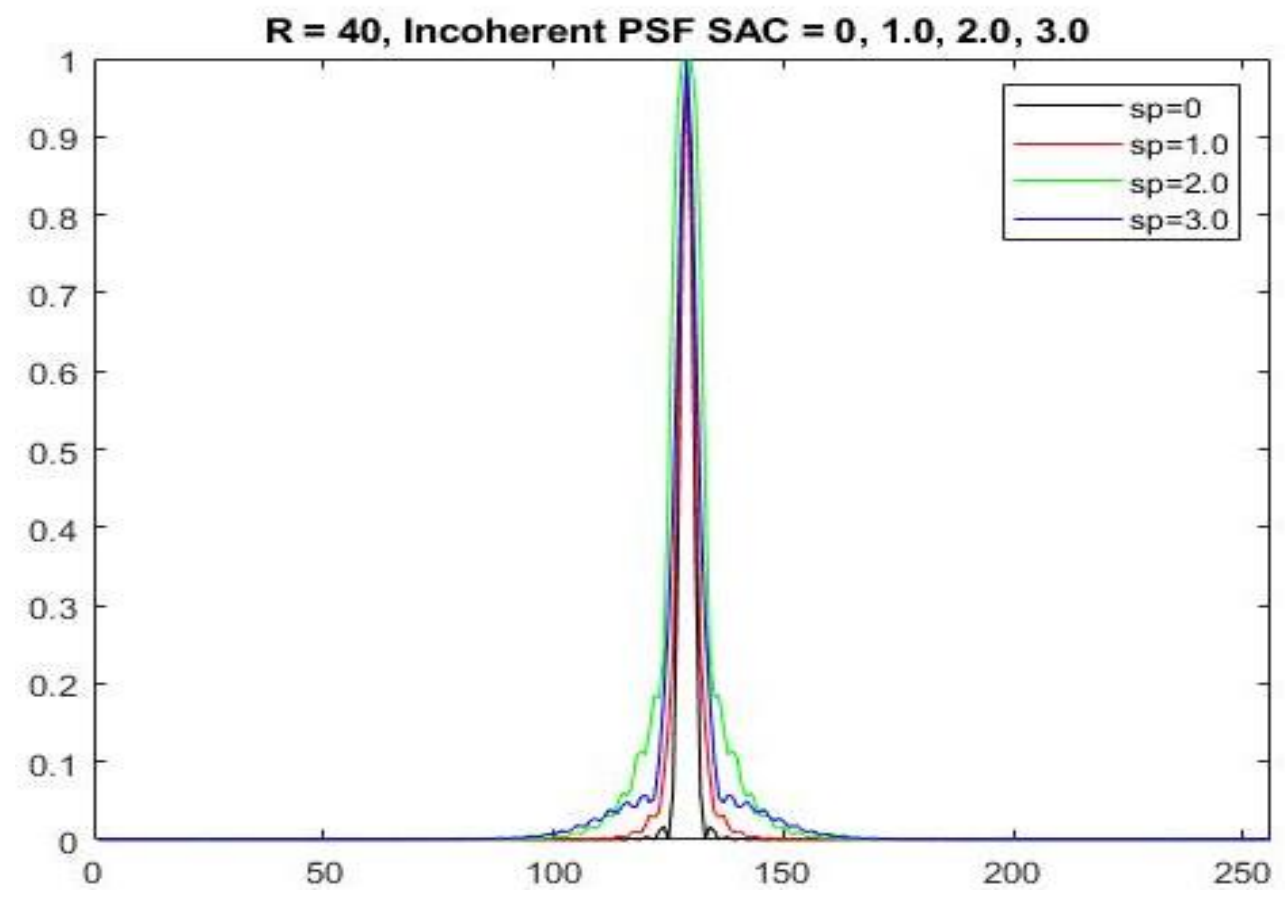

Figure 6-The PSF of the optical system in the case of incoherent illumination in the presence of spherical aberration when $\mathrm{R}=40$.

From Figures-(5) and (6), we found that PSF becomes wider (bad optical imaging system) under the effect of spherical aberration, especially for a small aperture. This is because of the blurring function produced when there is a spherical aberration.

Figure-(7) and Figure-(8) represent the effects of different magnitudes of spherical aberration $(\mathrm{M}=0,1$, 2 and 3) on MTF in the case of incoherent illumination for apertures with radii of $R=10$ and 40 , respectively. 


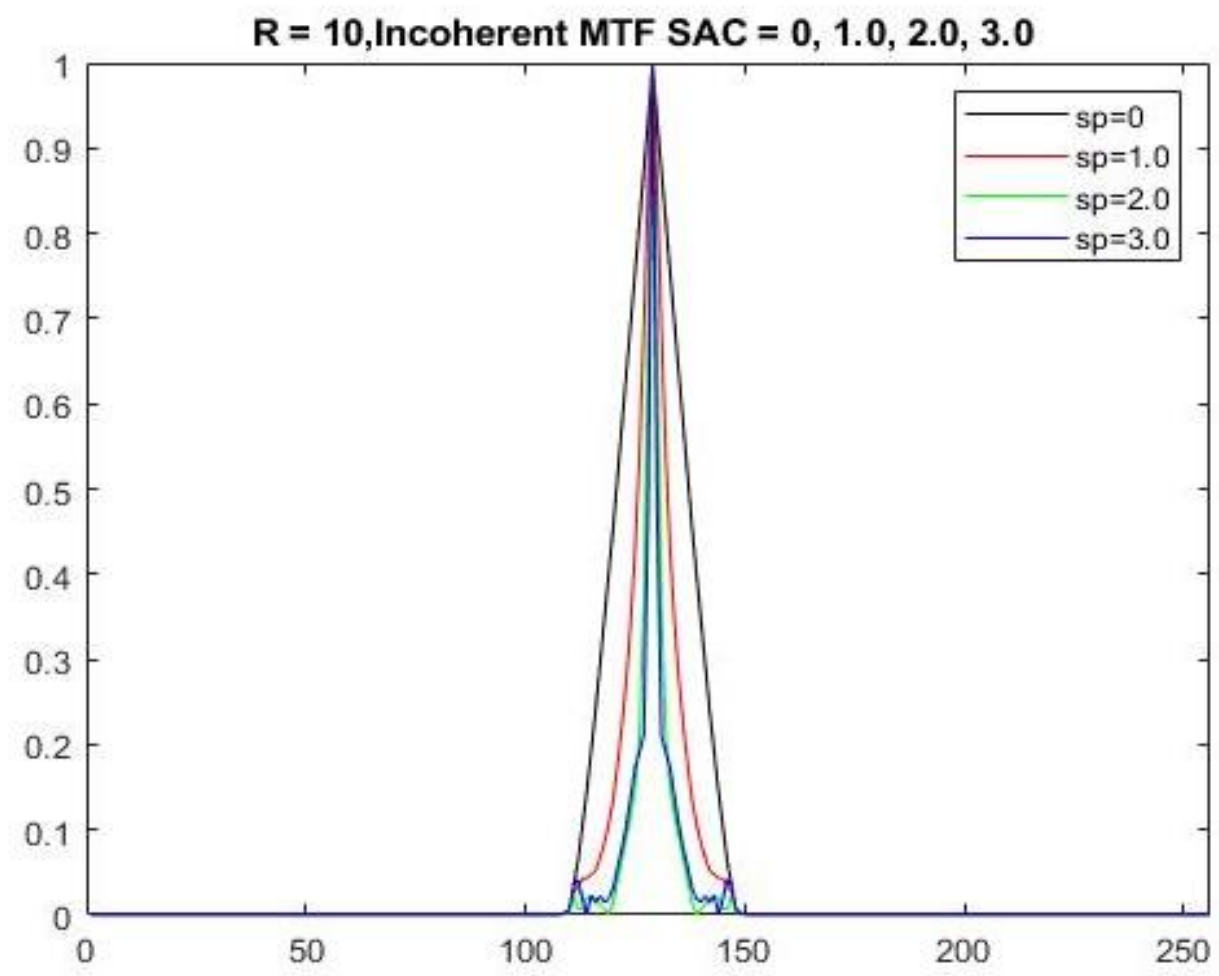

Figure 7-The MTF of the optical system in the case of incoherent illumination in the presence of spherical aberration when $\mathrm{R}=10$

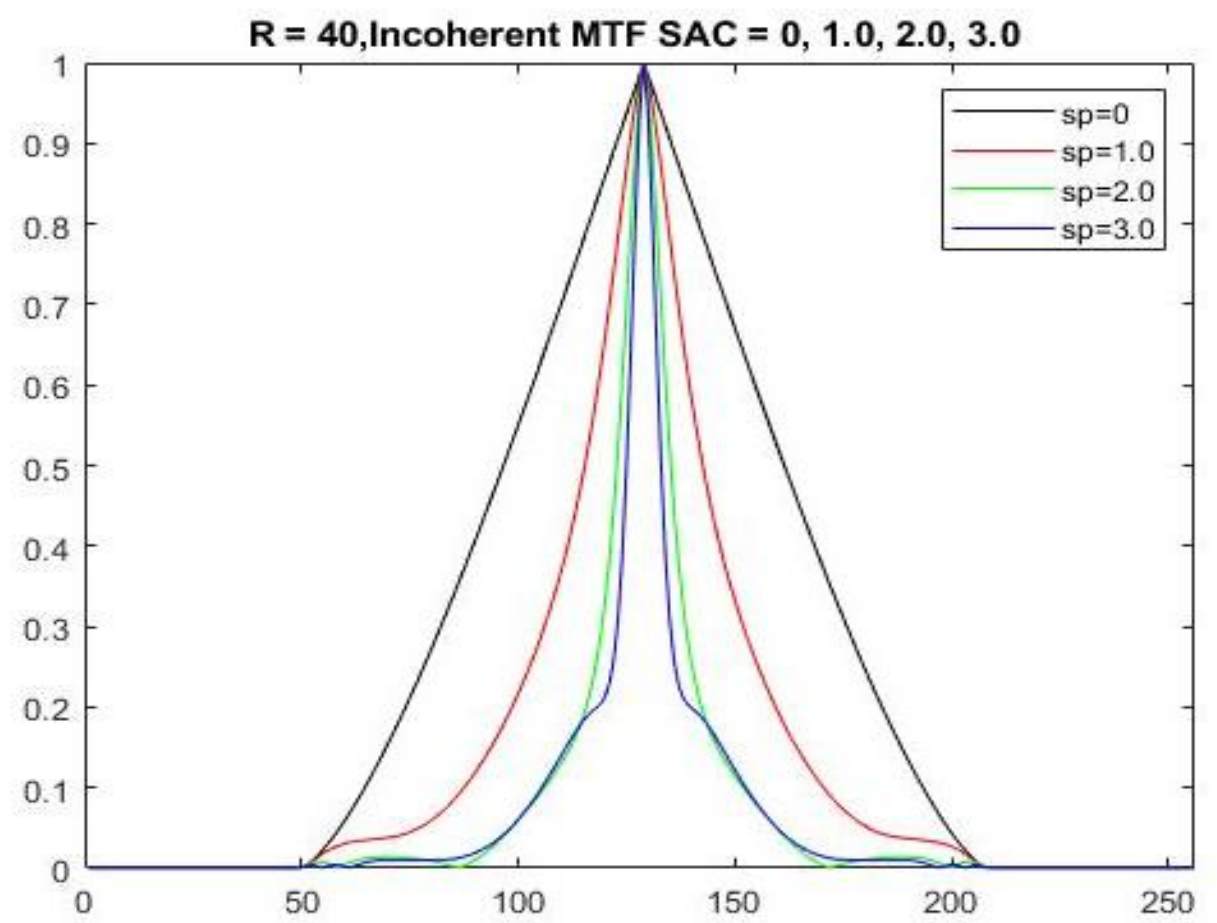

Figure 8-The MTF of the optical system in the case of incoherent illumination in the presence of spherical aberration when $\mathrm{R}=40$

From Figures-(7, 8), we found that MTF becomes narrower (bad optical imaging system) under the effect of spherical aberration, especially for a small aperture, which is because of the blurring function produced when there is a spherical aberration. 
Figure-(9) and Figure-(10) represent the effects of different magnitudes of spherical aberration $(M=$ $0,1,2$ and 3) on Point Spread Function in case of coherent illumination for apertures with radii of $\mathrm{R}=$ 10 and 40 , respectively.

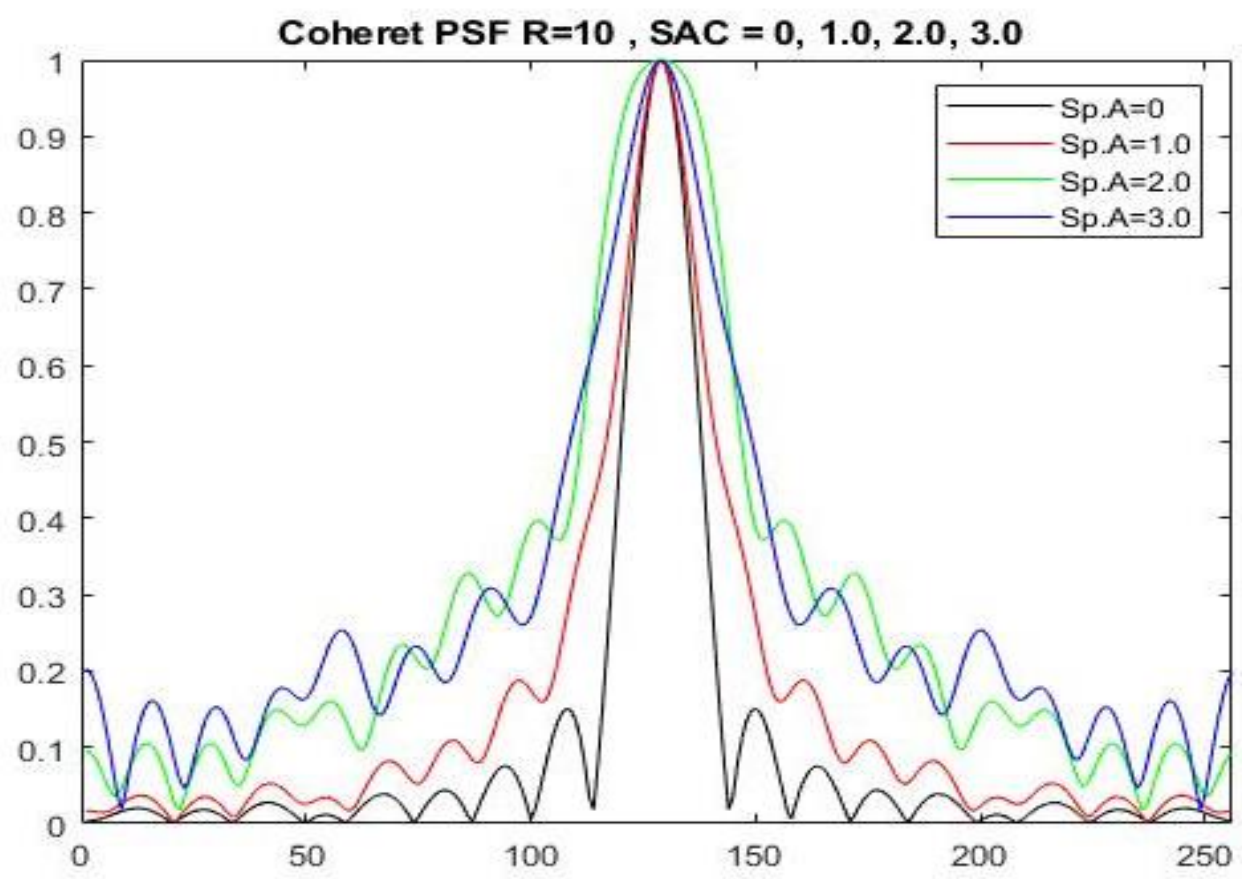

Figure 9-The PSF of the optical system in the case of coherent illumination in the presence of spherical aberration when $\mathrm{R}=10$

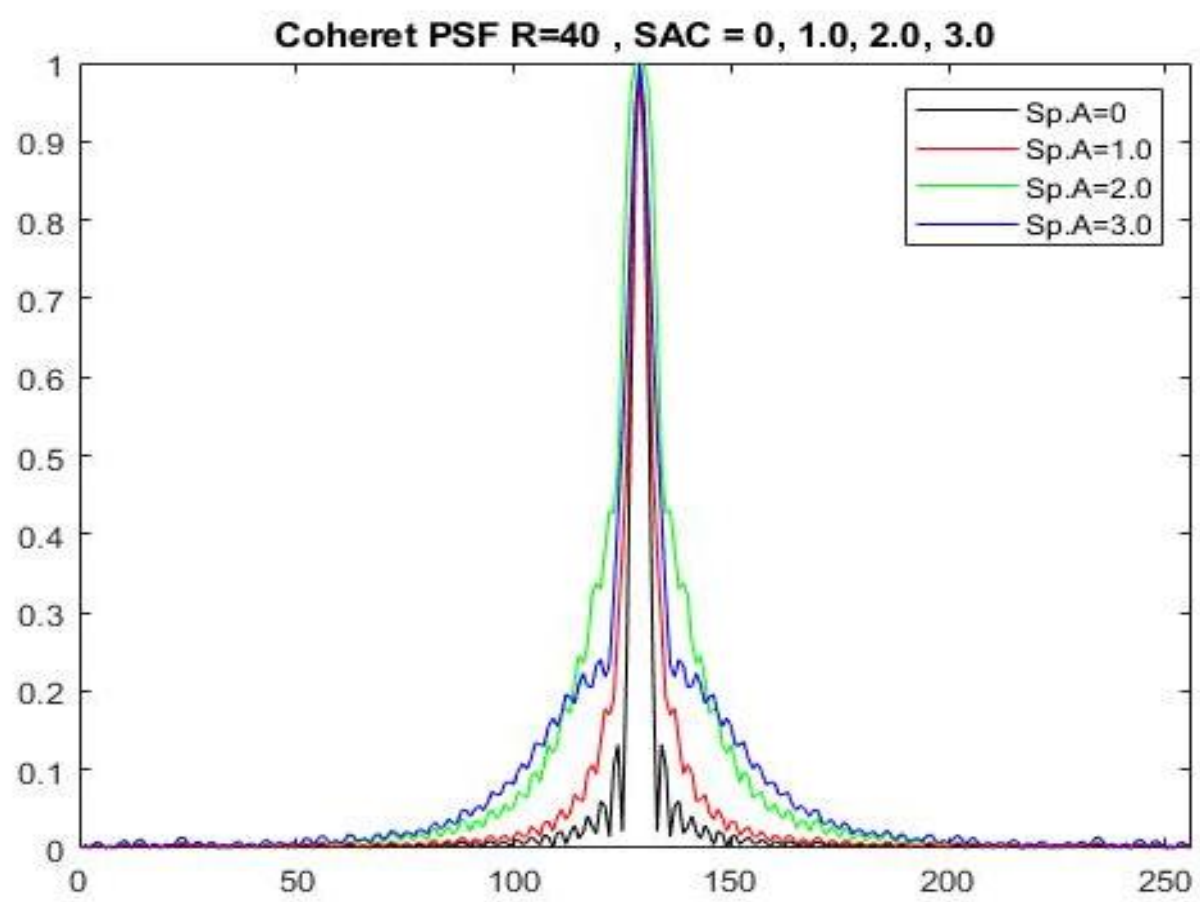

Figure 10-The PSF of the optical system in the case of coherent illumination in the presence of spherical aberration when $\mathrm{R}=40$

From Figures-(9, 10), we found that PSF becomes wider (bad optical imaging system) under the effect of spherical aberration, especially in the case of coherent illumination and small aperture. This is because of the blurring function produced when there is a spherical aberration and because of the phase of coherent illumination. 
Figure-(11) and Figure-(12) represent the effects of different magnitudes of spherical aberration $(\mathrm{M}=0,1,2$, and 3) on MTF in the case of coherent illumination for apertures with radii of $\mathrm{R}=10$ and 40 , respectively.

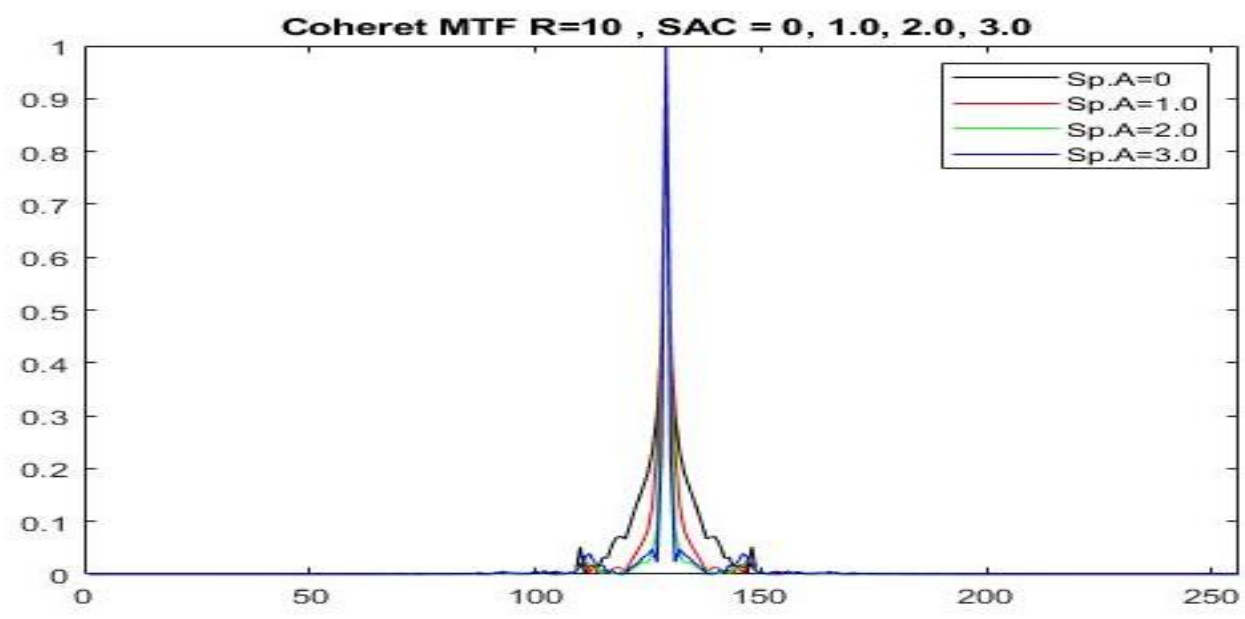

Figure 11-The MTF of the optical system in the case of coherent illumination in the presence of spherical aberration when $\mathrm{R}=10$

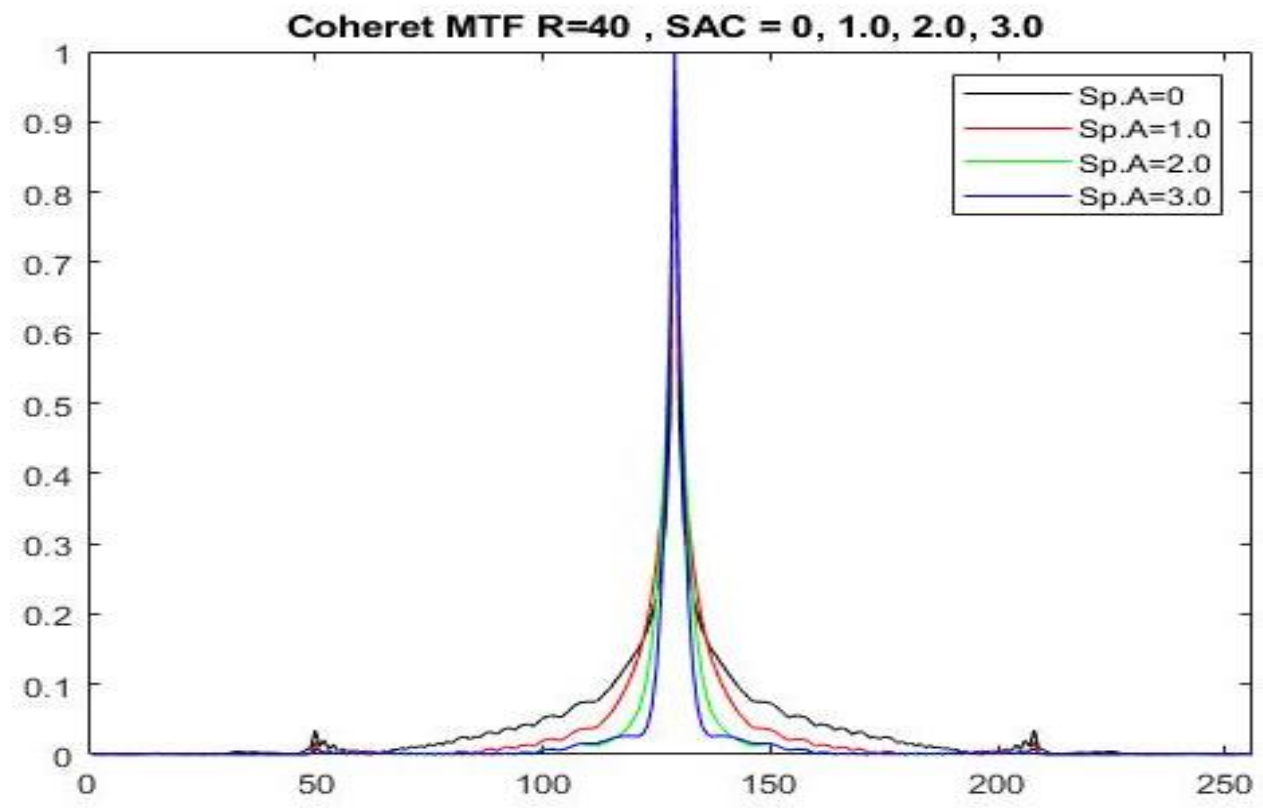

Figure 12-The MTF of the optical system in the case of coherent illumination in the presence of spherical aberration when $\mathrm{R}=40$

From Figures-(11) and (12), found that MTF becomes narrower (bad optical imaging system) under the effect of spherical aberration, especially in the case of coherent illumination and small aperture. This is because of the blurring function produced when there is a spherical aberration and because of the phase of coherent illumination.

From the above results we can conclude that imaging through incoherent illumination is better than that through coherent illumination and imaging through large aperture is better than that through small aperture, from the effect of spherical aberration imaging become gets worse while increasing $\mathrm{M}$ especially for small aperture and for coherent illumination because of the phase of spherical aberration and phase of coherent illumination.

To study quantitatively the effect of spherical aberration, in case of coherent and incoherent illumination and for different radii of aperture, we used the Root Mean Square error (RMSE) parameter, which is given by the equation: 


$$
\mathbf{R M S E}=\sqrt{\frac{\sum_{\mathrm{i}=\mathbf{1}}^{\mathrm{n}}\left(\mathbf{y}_{\mathrm{j}}-\mathbf{y}_{\mathrm{i}}\right)^{2}}{\mathbf{n}}}
$$

where $\left(y_{j}-y_{i}\right)^{2}$ is the Residuals difference between the actual values and the predicted values of aberration [14].

Figure-(13) and Figure-(14) represent the RMSE results on MTF due to the effects of different magnitudes of spherical aberration $(M=0,1,2$, and 3$)$ in the cases of coherent and incoherent illumination for apertures with radii of $\mathrm{R}=10$ and 40 , respectively.

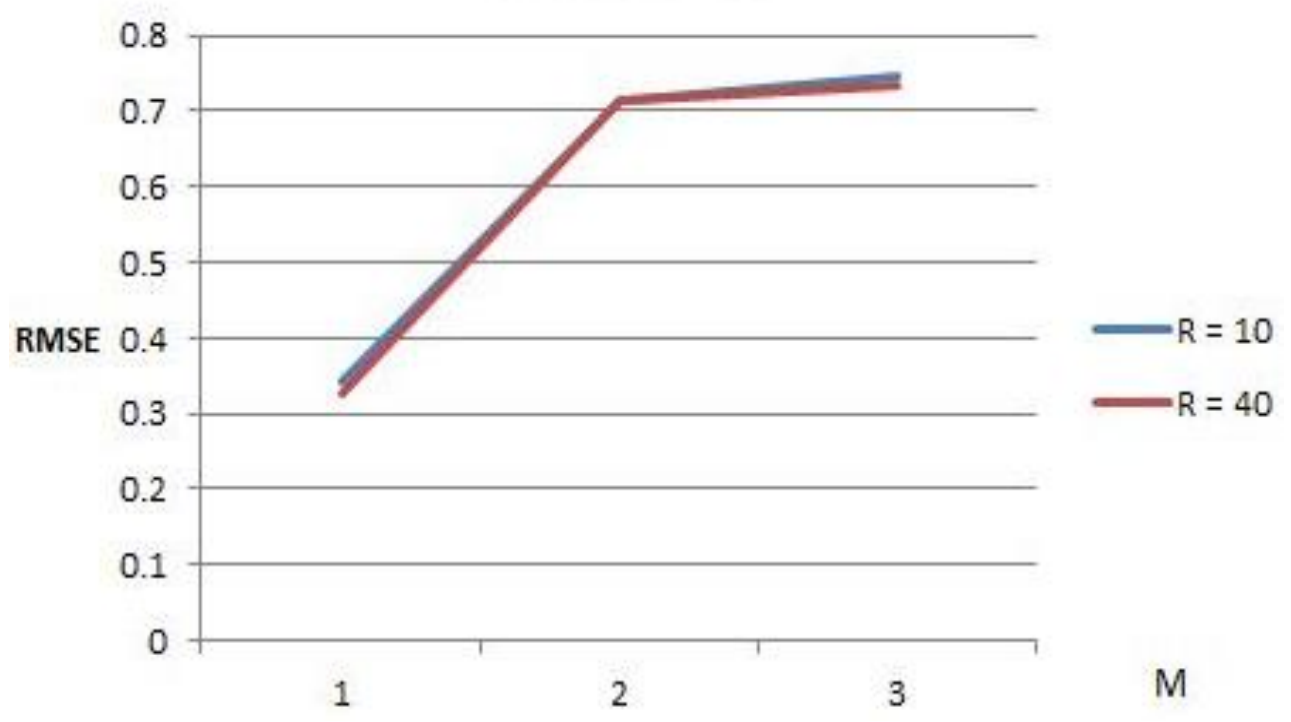

Figure 13-The RMSE of the optical system in the case of incoherent illumination in different magnitudes of spherical aberration when $\mathrm{R}=10$ and 40

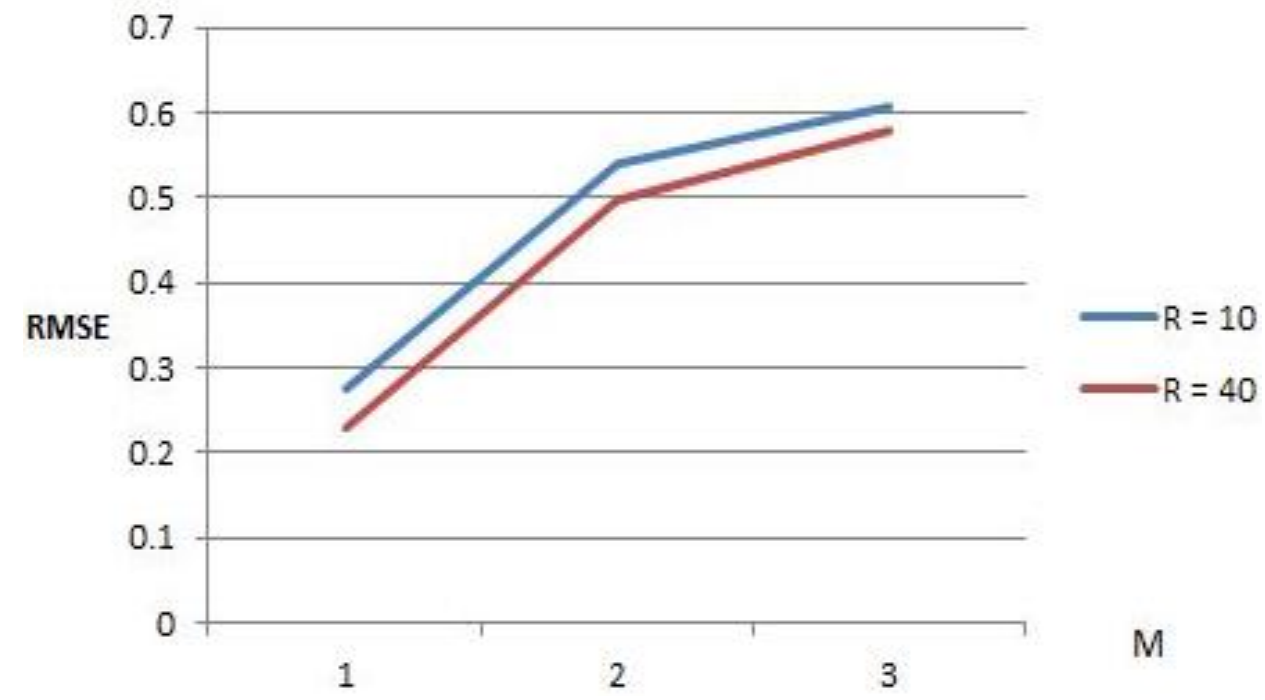

Figure 14-The RMSE of the optical system in the case of coherent illumination in different magnitudes of spherical aberration when $\mathrm{R}=10$ and 40

From Figures-(13) and (14), we found that RMSE is increased with increasing the spherical aberration coefficient (bad optical imaging), especially for small aperture and coherent illumination.

\section{Conclusions}

This work describes the effects of spherical aberration on the intensity of the point spread function and the modulation transfer function in the cases of incoherent and coherent illumination.

From the results, we can conclude that imaging through incoherent illumination is better than that through coherent illumination and imaging through large aperture is better than that through small 
aperture. From the effect of spherical aberration imaging become gets worse while increasing $\mathrm{M}$ especially for small aperture and for coherent illumination because of the phase of spherical aberration and phase of coherent illumination.

In addition, the root mean square error was increased with increasing $M$, which indicates worse optical imaging with increasing the spherical aberration coefficient.

We also observed that when the spherical aberration increase the intensity of the point spread function in case of incoherent and the amplitude of the PSF in case of coherent illumination increase but when the spherical aberration increase the intensity of the modulation transfer function in case of incoherent and amplitude in case of coherent decrease because the OTF $=$ Fourier transform of the PSF and the $\mathrm{MTF}=|\mathrm{OTF}|$ So it's take a real values only.

\section{References}

1. Ayad A. Al-Ani, 1995. "Restoration of atmospheric epically Degraded images" Ph.D. Thesis, dept. of physics, College of Science, Baghdad University

2. John K. 2008. "Journal of the Optical Society of America", 25: 159-17

3. Adnan Falih and Ban Hussein. 2013. "The Effect of Coma Aberration on Point Spread Function for Array Synthetic Circular Obscured Aperture" Journal of Kufa-Physics, 5(1).

4. Dereniak E. 2010. "OPTI 340-Optical Design. Lecture, the University of Arizona, Tucson, AZ, spring

5. Karamata B. and Lambelet P. 2004. "introduction to Fourier Optics" 29: 736-738

6. Safa Kamal Mustafa and Raied K. 2019. "Studying the Effect of Annealing on Optical and Structure Properties of optical system" Iraqi Journal of Science, 60(10): 2168-2176

7. Smith T. 2007. "Spherical Aberration in thin lenses". Scientific Papers of the Bureau of Standards. 18: $559-584$

8. Guenther R. 1990. "Modern Optics” Cambridge: John Wiley \& Sons Inc, p 130

9. Denis and Kathren and Samuylov 2019. "Modelling point spread function in fluorescence microscopy with a sparse Gaussian mixture: trade-off between accuracy and efficiency" university of poston, 12: 38-54

10. Mahmmod Talib and Nawal Mohammed, 2019." Gene Polymorphisms Impacts on Its Serum Levels in Prostate Cancer Iraqi Patients" Iraqi Journal of Science, 60(6): 1188-1196

11. Goodman, 1998. "introduction to Fourier optics" megraw hill New York

12. Arreola L. 2015. "plane vibrations of a rectangular plate: Plane wave expansion modelling and experiment", Journal of Sound and Vibration, 342: 168-176

13. Afleck B. "Born and Wolf 1970. "Principal of optics" pergaman London

14. Susan H. 2012. "Regression Effect and Regression", Journal of the Optical Society of America. 48: $193-213$ 